\title{
A New Hybrid METHOd FOR THE SEGMENTATION OF THE BRAIN MRIS
}

\author{
Mohamed Zaki Abderrezak, Mouatez billah Chibane, Prof. Karim Mansour \\ Laboratory of Study of Electronic Materials for Medical applications \\ University Constantine 1 - Road of Ain El Bey - Constantine - Algeria.
}

\begin{abstract}
The magnetic resonance imaging is a method which has undeniable qualities of contrast and tissue characterization, presenting an interest in the follow-up of various pathologies such as the multiple sclerosis. In this work, a new method of hybrid segmentation is presented and applied to Brain MRIs. The extraction of the image of the brain is pretreated with the Non Local Means filter. A theoretical approach is proposed; finally the last section is organized around an experimental part allowing the study of the behavior of our model on textured images. In the aim to validate our model, different segmentations were down on pathological Brain MRI, the obtained results have been compared to the results obtained by another models. This results show the effectiveness and the robustness of the suggested approach.
\end{abstract}

\section{KEYWORDS}

Hybrid Segmentation, Petreatment, Cerebral.MRI, Multiple Sclerosis, tumors.

\section{INTRODUCTION.}

The segmentation plays a big role in the image processing. It is carried out before the stages of analysis and catch of the decisions in several image treatments of, such as the detection of the cerebral anomalies in the magnetic resonance images.

During these last years several methods of segmentation were developed $[1,2,3,4,5,6]$. among them active contours (ACM) which was applied to various types of medical imaging modalities. Generally in the literature, we distinguishe two types of (ACM): model based contour $[1,2,24]$ and model based area $[3,4,5,6]$. A good review of the principal models of active contours can be found in the two following references $[7,8]$.

To locate the areas borders in the segmentation based contour, we can use the gradient information. This method allows the detection of the transitions between the various areas of the image $[1,2,3]$. The detectors of contours used can be simple, like the operators of Sobel and Roberts [9], or more complexes such as the operator of Canny [11]. The results of this segmentation are the candidates of the limits of the image objects. However, they can present false detections because its models remain sensitive to the noises.

The principle of the segmentation based area is to take into account some descriptors of areas, such as the intensity, the color, the texture or the movement [13]. The function to be minimized in this case, is written like an integral on the area and not on contour. However, the areas obtained do not correspond in all cases, with the objects represented in the image. The limits of the obtained areas are usually vague and do not coincide exactly with the bornes of the objects of the image (Kermad and Chehdi, 2002) [14]. 
Signal \& Image Processing : An International Journal (SIPIJ) Vol.5, No.4, August 2014

One of the most used methods of segmentations based area in imaging, is the model of Chan and Vese [3]. It had much success on binary images with the assumption that each area in the image, is homogeneous. While on medical images, the same area is found with various degrees of homogeneities, from where multiple modifications made to the model of Chan and Vese in order to adapt it to different type of medical imaging modalities [15].

Another approach of segmentation called hybrid segmentation, combining the advantages of the two preceding ones, was developed [16,17]. The method based area, aims at the detection of the ROI (limits of the regions of interest), and uses the segmentation based contour to determine the limits of these areas. The algorithms combining the techniques of segmentation based on the areas and those based on contours take advantage of the complementary of the information on the area and contour [18]. Each step is executed iteratively allowing the other to improve. Yu et al. [19] fused methods by set of level (implicit deformable models) with Bayesian techniques to segment the cortex. Yang and Kruggel [20] combine Bayesian approaches with Watershed algorithms to segment the cortical sulci.

Our work concerns the development of a new hybrid approach starting from a geodetic active contour. This approach is tested on cerebral images obtained by magnetic resonance. In our model the gradient is replaced by the GVC (Gradient Vector Convolution) to widen the card of capture.

\section{MATERIAL AND Method.}

The problem of the detection of the artifacts in the medical images hasn't a single solution. It is necessary however, to choose most adequate for the images obtained by MRI which are affected by a random noise which can deteriorate the segmentation. Before carrying out detection, we have developed a new algorithm to eliminate all the parts around the brain which present little interest, even not interest for the physician (example skin of the head).

Step 1: to calculate the variance in various scales and various directions with an aim of extracting a feature set of the initial image knowing that:

$$
\operatorname{var}(\mathrm{x})=\sum_{\mathrm{n}} \frac{\mathrm{x}_{\mathrm{i}}-\overline{\mathrm{x}}}{\mathrm{n}}
$$

Step 2: to calculate the gradient and the coefficient of conduction.

Step 3: to use the algorithm of Otsu to obtain a binary image, and to benefit from the level of gray of the cranium, which is darker than the cerebrospinal fluid which surrounds the brain.

Step 4: To carry out a morphological opening using a disc of ray 5 pixels on the binary image, which makes it possible to eliminate the smaller related objects.

Step 5: To fill the parts isolated from the brain not eliminated at the time of the preceding stage.

Step 6: To superimpose the two images to make the extraction.

Step 7: Final image.

To eliminate the artifacts from the image, we apply a Non Local Means filter, which proved its efficiency on the MRI [10]. Being given the image using method NLM the value filtered in a point is calculated like a weighted average of all the pixels of the image given by:

$$
\begin{aligned}
& \operatorname{NLM}(u(x))=\sum_{y \in \Omega} w(x, y) \cdot u(y) \\
& 0 \leq w(x, y) \leq 1, \sum_{y \in \Omega} w(x, y)=1
\end{aligned}
$$


Signal \& Image Processing : An International Journal (SIPIJ) Vol.5, No.4, August 2014

$w(x, y)$ represents the NL-means weights according to the similarity between the values of the vicinities and are written:

$$
\begin{aligned}
w(x, y) & =\frac{1}{Z(x)} e^{-\frac{d(x, y)}{h^{2}}} \\
Z(x) & =\sum_{y \in \Omega} e^{-\frac{d(x, y)}{h^{2}}}
\end{aligned}
$$

$h$ a parameter which allows the control of the filter, represents a constant of standardization, and d the Euclidean distance which is written:

$$
d(x, y)=G_{x}\left\|Y\left(N_{x}\right)-Y\left(N_{y}\right)\right\|_{R_{\text {sim }}}^{2} \text { avec } R_{\text {sim }} \in[1.5]
$$

The obtained results (figure 2) show the effectiveness of our algorithm to carry out the extraction of the brain image in the aim to prepare it to the segmentation.

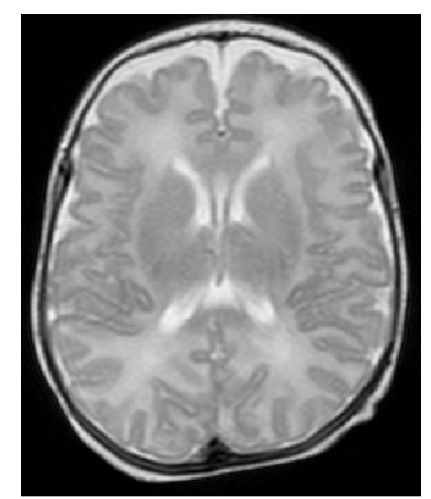

Initial Image

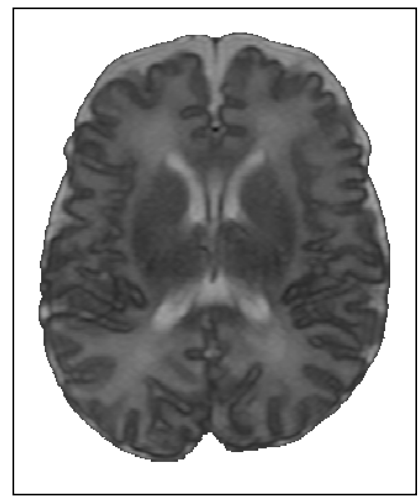

final Image

Figure 2. Pretreatment of a MRI with our algorithm

When the pretreatment is finished, we carry out the segmentation by using the model of geodetic contours proposed by Casselles et al. [21] and given by the following expression

$$
\frac{\partial \phi(\mathrm{x}, \mathrm{y}, \mathrm{t})}{\partial \mathrm{t}}=\mathrm{g}(|\nabla \mathrm{u}|)(\mathrm{k}+\mathrm{v})|\nabla \emptyset(\mathrm{x}, \mathrm{y}, \mathrm{t})|+\nabla \mathrm{g}(\nabla \mathrm{u}) \cdot \nabla \emptyset(\mathrm{x}, \mathrm{y}, \mathrm{t})
$$

The gradient allows the detection of the borders, but this model remains ineffective when these borders are fuzzy. To solve this problem, Chan and Vese [3] have proposed a geometrical segmentation ACM based on the model of Munmford and Sahn [22]. This model starts with the application of an initial contour in the field of the image for then evolving/moving and stopping at the borders of the objects. The function of energy defined by Chen and vese is written:

$$
\begin{gathered}
I: \Omega \rightarrow \mathrm{R} \\
x \in \Omega \\
E^{c v}\left(C, c_{1}, c_{2}\right)= \\
\mu . \text { length }(c)+\text { v.area }(\text { inside }(c))+\lambda_{1} \int_{\text {inside }(c)}\left(I-C_{1}\right)^{2} d x+ \\
\lambda_{2} \int_{\text {outside }(c)}\left(I-C_{2}\right)^{2} d x
\end{gathered}
$$

Where $\mu \geq 0, v \geq 0, \lambda_{1}, \lambda_{2}>0$ represent positive constants. 
$C_{1}, C_{2}$ represent two constants which define the approximation of the intensity respectively inside and outside contour $\mathrm{C}$.

After minimization of the function (5) Chan and Vese obtain the following formula:

$$
\frac{\partial \emptyset}{\partial t}=\delta(\emptyset) \cdot\left[\mu \cdot k-v-\lambda_{1}\left(I-C_{1}\right)^{2}+\lambda_{2}\left(I-C_{2}\right)^{2}\right]
$$

Where $\delta(\phi)=H_{\epsilon}^{\prime}(x)=\frac{1}{\pi} \cdot \frac{\epsilon}{\varepsilon^{2}+x^{2}}$ is the Dirac function.

$K$ represents the function of curvature, calculated with the following equation:

$$
\begin{gathered}
K=\operatorname{div}\left(\frac{\nabla \emptyset}{|\nabla \phi|}\right) \\
C_{1}(\varnothing)=\frac{\int_{\Omega} \mathrm{I} \cdot \mathrm{H}(\varnothing) \mathrm{d} \emptyset}{\int_{\Omega} \mathrm{H}(\emptyset) \mathrm{d} \emptyset}, \quad C_{2}(\varnothing)=\frac{\int_{\Omega} I(1-H(\emptyset)) \partial \emptyset}{\int_{\Omega}(1-H(\emptyset)) \partial \emptyset}
\end{gathered}
$$

The term $-\lambda_{1}\left(\mathrm{I}-\mathrm{C}_{1}\right)^{2}+\lambda_{2}\left(\mathrm{I}-\mathrm{C}_{2}\right)^{2}$ plays an important role in the evolution of initial contour. In our model, $v=0$ in the aim to decrease the time of calculates.

In this model, Chan and Vese use the Dirac function $\delta(\phi)$ to carry out the regularization. In our model, this term is replaced by $|\phi(x, y)|$ in order to widening the range of capture. The new function is then written in the following form:

$$
\frac{\partial \emptyset}{\partial t}=|\nabla \phi| \cdot\left[\mu \cdot k-\lambda_{1}\left(I-C_{1}\right)^{2}+\lambda_{2}\left(I-C_{2}\right)^{2}\right]
$$

We suppose that $\lambda_{1}=\lambda_{2}=\lambda_{0}$ and by summoning it with the geodetic model of the expression (4), we obtain the following function:

$$
\frac{\partial \emptyset}{\partial t}=|\nabla(\varnothing)| \cdot\left[g(k)+\gamma\left(C_{1}-C_{2}\right)\left(u+\frac{C_{1}+C_{2}}{2}\right)\right]+\nabla g \cdot \nabla \emptyset
$$

$\gamma$ is a constant which ensures the proportionality between the term of the forces of gradient and the term of the forces of area. $g$ represents the function of stop which is calculated by the following expression :

$$
\mathrm{g}=\frac{1}{1+\left|\nabla \mathrm{G}_{\sigma} * I\right|^{\mathrm{p}}} \quad \text { with } \quad p \geq 1
$$

Where $G_{\sigma}$ is the Gaussian core. The second term of the equation was replaced by term representing the GVC (Gradient Vector Convolution) [23], to regulate the problem of the dependence to the initialization of contour or to obtain a contour which is able to be driven to the border of the regions of interest.

The approximation of the equation (10) is then written in the following form:

$$
\emptyset_{\mathrm{i}, \mathrm{j}}^{\mathrm{n}+1}+\Delta \text { t. }\left[|\nabla(\emptyset)|\left(\text { g. } \nabla \mathrm{u} .(\mathrm{k})+\gamma\left(\mathrm{c}_{1}-\mathrm{c}_{2}\right)\left(\mathrm{u}+\frac{\left.\mathrm{c}_{1}+\mathrm{c}_{2}\right)}{2}\right)\right)+\mathrm{w}_{\mathrm{g}} \cdot \mathrm{w}_{\varnothing}\right]
$$

$c_{1}, c_{2}$ are performed from the function (8).

$\gamma$ is a positive constant.

$\Delta \mathrm{t}$ the step between each iteration.

$\left(\mathrm{w}_{\mathrm{g}} \cdot \mathrm{w}_{\emptyset}\right)$ represent GVC. 
Signal \& Image Processing : An International Journal (SIPIJ) Vol.5, No.4, August 2014

To carry out the segmentation, we have used the following algorithm:

Step $1: \emptyset_{0}(i, j)$ initial levels set contour.

Step $2: u(i, j)$ image to be segmented.

Step $3:[\mathrm{cl}, \mathrm{cn}]$ size of the image.

Step $4:$ Constants $(\gamma, \Delta t, \sigma)>0$

Step 5 : Initialization of the function level set: $\emptyset_{0}(i, j)=\emptyset(i, j)$

Step 6 : Calculation of the gradient $\mathrm{g}$ :

For $i=1$ to $\ln$

For $j=1$ to $C n$ do

$$
g(|\nabla u(i, j)|)=\frac{1}{1+\left|\nabla G(i, j)_{\sigma} * u(i, j)\right|}
$$

Step 7 : Calculation of the term of curvature K:

For $i=1$ to $\ln$

For $j=1$ to $C n$ do

$$
k(i, j)=\operatorname{div}\left(\frac{\nabla \emptyset(i, j)}{|\nabla \emptyset(i, j)|}\right.
$$

Step 8: Calculation of $c_{1}, c_{2}$ one using the function (8).

Step 9: Calculation of $w_{g} \cdot w_{\phi}$ the Gradient Vector Convolution.

Step 10 : Calculation of $\phi^{+1}(i, j)$

$$
\emptyset_{i, j}^{n+1}+\Delta t .\left[|\nabla(\emptyset(i, j))|\left(g .(k)+\gamma\left(c_{1}-c_{2}\right)\left(u(i, j)+\frac{\left.c_{1}+c_{2}\right)}{2}\right)\right)+w_{g} \cdot w_{\varnothing}\right]+\emptyset^{t}(i, j)
$$

Step 11 : Outputs, $\emptyset_{i, j}^{n+1}(i, j)$ is the final contour of the level-set segmented.

The model suggested was implemented on a platform Matlab R2013a under Windows@ 7 Professional, with a processor Intel® Core ${ }^{\mathrm{TM}} \mathrm{i} 3-2.30 \mathrm{GHz}$, and 4.00 Go of RAM

\section{RESULTS.}

The method suggested was tested on a textured image rough the stage of pretreatment with the filter Non local Means. We have fixed the parameter of regularization $\mu$ at 0.5 , the step of time $\Delta t$ to 0.01 and we have varied the constant of proportionality " $\gamma$ " until finding balance between internal and external forces for a good evolution of the curve. After several tests $(\gamma=0.5)$ figure 3 shows the result of the segmentation of our model on the selected image.

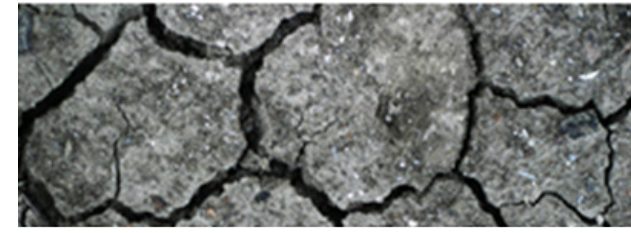

Image textured before segmentation

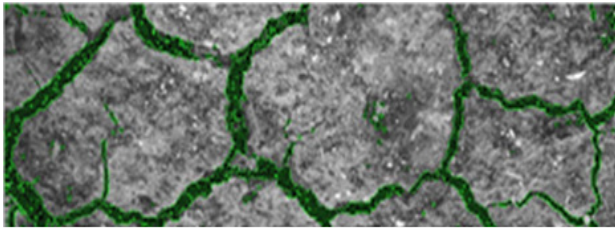

Textured image after segmentation

Figure 3. Segmentation of a textured image rough 160 iterations

Then we have tested our model on pathological MRI images which represent patient with brain tumor or with multiple sclerosis. Figure 4 illustrates the results obtained by our method on this image. We have compared them to these obtained by the Chan and Vase model [3]. 
Signal \& Image Processing : An International Journal (SIPIJ) Vol.5, No.4, August 2014

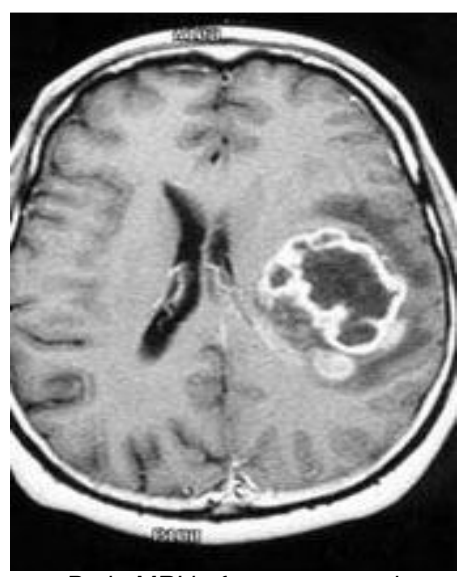

Brain MRI before segmentation

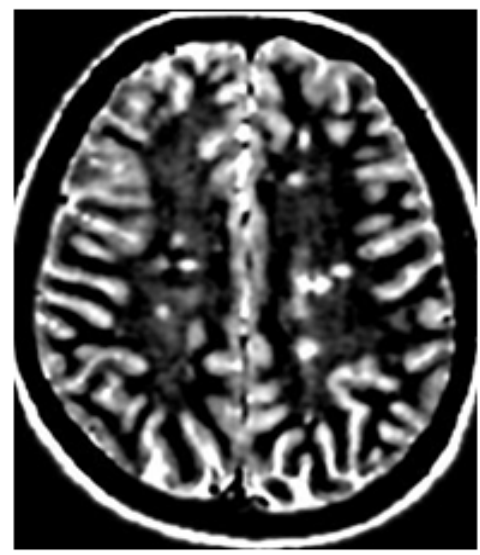

Brain MRI before segmentation

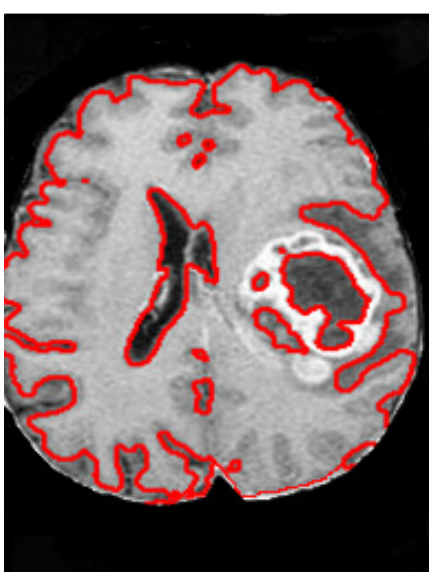

Segmentation with rough model C-V 200 iterations

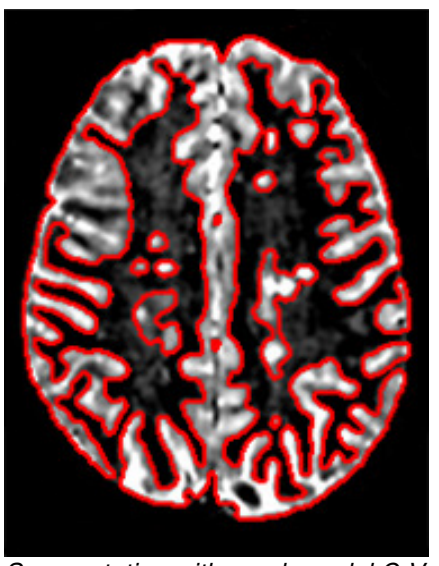

Segmentation with rough model C-V 300 iterations

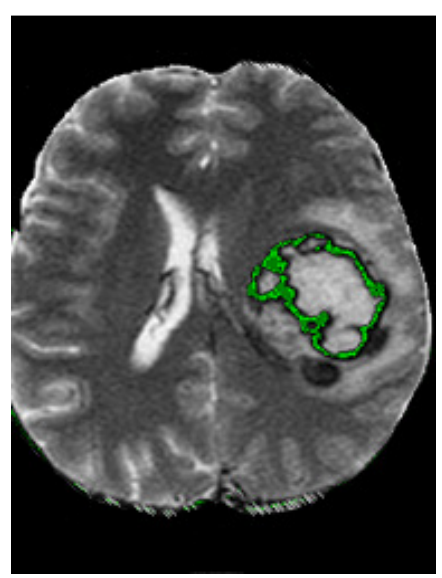

MRI rough segmentation with our model $(\mu=0.5, \Delta T=0.02, \gamma=0.01$ and sigma $=1.5$

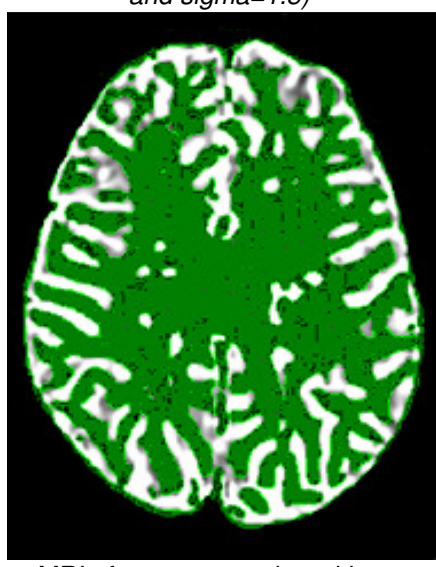

MRI after segmentation with our model $(\mu=0.5, \Delta T=0.02, \gamma=0.01$ and sigma $=0.5$ )

Figure 4. Comparison between our model and the Chan and Vese model

According to the results obtained in figure 4, we note that the quality of our segmentation is good for tumor and for multiple sclerosis detection. Indeed, the anomalies are detected and contours are well closed.

The comparison between our model and the Chan and Vese approach [3] shows effectiveness of our formula on the Brain MRI. We remark that our model is more precise for the determination of the true limit of the object, whereas the $\mathrm{C}-\mathrm{V}$ model has failed in the segmentation of the image with the brain tumor (figure 4). Indeed, after 200 iterations in the Chan-Vese model, the limit of the tumor is not detected. While when our model is used, the limit of the tumor is well detected after 80 seconds (figure 4 ).

We have noted that on the image 4.e (patient reached of a multiple sclerosis segmented by 600 iterations using the Chan and Vese approach, the limits of the spots affecting the white substance of the brain which represents the sclerosis lesions are not detected. On the other hand, our model (image4.f) the lesions in the white substance are detected with a good precision.

For the preprocessing of these images, we carried out the extraction of the brain image to reduce the calculation time, than we used a Non Local Means filter which shows its efficiency to decrease the noise present in the MRI images while preserving the quality of the image's contours 
Signal \& Image Processing : An International Journal (SIPIJ) Vol.5, No.4, August 2014

[10]. For the step of segmentation, we used a new geodetic active contours method. The obtained results on the MRI images show that our function is more adapted to this image type than the Chan and Vase model [17]. The experimental results on the MRI images show the efficiency and the robustness of our function. The short segmentation time of the images gives another advantage for the clinical use.

Thus, the uses of the GVC [23] give more robustness for our model to detect the different regions in our images using the strong gradients which correspond to the borders of these regions, while remaining insensitive to the initialization step. In contrast to the method of Chan and Vese which isn't able to extract the borders of cerebral pathologies with precision. These make our model more adapted to the MRI images processing.

\section{Conclusion.}

The contributions of this article are intended to build a model for automatic segmentation tumors and multiple sclerosis. We proposed a new model based segmentation combining region and edge-based segmentation. The results show the efficacy of the proposed model for the localization and the detection of the shape and size of the lesions present in the brain image. Further efforts are underway to improve the execution time of the program to allow us to to Implement the program in the machine and provide more tools for the detection of the tumors and multiple sclerosis.

\section{REFERENCES.}

[1] S.Osher, J.Sethian, "Faces propagating with curvature depend speed: Algorithms based one Hamilton-Jacobi formulation" newspaper off computational Physic 79, p 12-49, 1988.

[2] C.Li, C.Xu, C.Gui and M.D.Fox, "Level set evolution without Re-initialization: has new variational formulation" in Proc IEEE, Conference one Computer Vision and Pattern Recognition, vol. 01, p 430-436, 2005.

[3] T.Chan and L.Vese, "Activates contours without edges" IEEE Transaction one Processing image, vol. 10, No 2, p 266-277, Feb 2001.

[4] B.Vemuri and Y.Chen, "Joined image registration and segmentation" Geometric Level Set Methods In Imaging, and Graphics, pp.251-269, 2003.

[5] R. Ronfard, "Area-based strategies for activates contour model", International. Joural off Computer. Vision, 13:2, p 229-251, 1994.

[6] L. Wang, C. Li, Q. Sun, D. Xia, C. Kao, "Brain local MR. image segmentation using and total intensity fitting activates contours/surfaces off", in Proceedings Medical Image Computing and Computer Aided Intervention, Lecture Notes in Computer Science, vol. 5241, Part I, Springer, Berlin, pp. 384-392, 2008

[7] L. He, Z. Peng, B. Everding, X. Wang, C. Han, K. Weiss, W. Wee, "has comparative study off deformable medical contour methods one image segmentation", Image and Vision Computing. Volume 26. P 141-163. February 2008.

[8] Jain, Y. Zhong, Mr. Dubuisson-Jolly, "Deformable template models has review”, Signal Process. Volume 71 (2). P 109-129, December 1998.

[9] H. Khosravi, E. Kabir," Farsi make to recognition based one Sobel - Roberts features”, Pattern Recognition Letters. Flight 31, pp.75-82, January 2010.

[10] Manjon JV, Carbonell-Caballero J, Lull JJ, Garcia-Martí G, Marti-Bonmati L, Robles Mr. "MRI denoising using Means Not-Room”. Medical Analysis.pp Image 514-523. Aug 2008.

[11] Canny, J.F. (1986). "Computational Approach to edge detection has". Pattern Analusis and Machine Intelligence, IEEE Transactions, p 679-698, Nov. 1986.

[12] Gonzalez, R.C and Woods, R.E. "DIGITAL Image Processing using MATLAB". 2nd edition, Prentice Hall, 2009.

[13] A. Tsai, A. Yezzi, A.S. Willsky, "Curve evolution implementation off the Mumford-Shah functional for image segmentation, denoising, interpolation, and magnification", Trans IEEE. 
Signal \& Image Processing : An International Journal (SIPIJ) Vol.5, No.4, August 2014

Image Process. Volume 10,1169-1186. Aug 2001.

[14] Kermad, C.D. and Chehdi, K. "Automatic image segmentation system through iterative edgearea co-operation”. Image and Computing Vision, flight 20, p 541-555. June 2002.

[15] L. Vese, T. Chan, "have multiphase level set framework for image segmentation using the Mumford and Shah model", Internatiola Journal off Computer Vision 50 (3). P 271-293. 2002.

[16] Bellet, F., Salotti, Mr. and Garbay, C. "an opportunist and co-operative approach for the vision of low-level". Processing of the signal, vol. 12, n 5, p. 479-494, (1995).

[17] Bertolino, P. and Montanvert, A. "Co-operation areas contours multirésolution in segmentation of image". Congress AFCET/Pattern recognition and artificial intelligence, Rennes, P. 299-307. January 1996.

[18] Zhang, Y.J. "Year Overview off Image and Video Segmentation in the Last 40 Years". Year Overview off Image and Video Segmentation 1. MRI Near. P 1-16. 2006.

[19] Yu, Z.Q., Zhu, Y., Yang, J., Zhu, Y.M., 2006. "Hybrid area-boundary model for cerebral cortical segmentation in MRI has". Medical Computerized Imaging and Graphics 30 (3), 197 208. April 2006.

[20] Faguo Yang, Kruggel Frithjof. "Automatic segmentation off human brain sulci”. Medical Image Analysis 12 (4), 442-451. Aug 2008.

[21] V.Caselles, R. Kimmel, and G. Sapiro, "Geodesic activate contours", International Newspaper off computer Vision V.22, No 1, pp.61-79, 1997.

[22] D. Mumford, J. Shah, "Optimal approximations by piecewise smooth functions and associated variational problems", Pure Communication one Applied Mathematics. P 577-685. 1989

[23] Yuanquan wang, Ynde Jia, "external force for activates contours: Gradient Vector Convolution", international conference one artificial intelligence, p 466-472. 2008.

[24] Khaldi Amine,Merouani Hayet Farida, "An active contour for range image segmentation", International Journal (SIPIJ) Vol.3, No.3, June 2012. 